Marquette University

e-Publications@Marquette

College of Nursing Faculty Research and

Publications

Nursing, College of

$9-5-2021$

\title{
Telehealth and the Psychiatric Mental Health Nurse Practitioner: Beyond the COVID-19 Pandemic
}

Jessica L. Zemlak

Patty Wilson

Brigit VanGraafeiland

Tamar Rodney

Follow this and additional works at: https://epublications.marquette.edu/nursing_fac

Part of the Nursing Commons 
Marquette University

e-Publications@Marquette

\section{Nursing Faculty Research and Publications/College of Nursing}

This paper is NOT THE PUBLISHED VERSION.

Access the published version via the link in the citation below.

Journal of the American Psychiatric Nurses Association, Online prior to print (September 5, 2021). DOI. This article is (C) SAGE Publications and permission has been granted for this version to appear in ePublications@Marquette. SAGE Publications does not grant permission for this article to be further copied/distributed or hosted elsewhere without the express permission from SAGE Publications.

\section{Telehealth and the Psychiatric Mental Health Nurse Practitioner: Beyond the COVID-19 Pandemic}

Jessica L. Zemlak

Johns Hopkins School of Nursing, Baltimore, MD

Patty Wilson

Johns Hopkins School of Nursing, Baltimore, MD

Brigit VanGraafeiland

Johns Hopkins School of Nursing, Baltimore, MD

Tamar Rodney

Johns Hopkins School of Nursing, Baltimore, MD

\section{Abstract}

Objective:

Examine the advantages, disadvantages, and challenges of telehealth for the psychiatric mental health nurse practitioner (PMHNP) in practice and student education. Describe areas for future research and 
policy development regarding telehealth in PMHNP practice and training during and beyond the COVID-19 pandemic.

\section{Methods:}

Review current evidence, standards of practice, and education for the PMHNP.

Results:

Recent rapid pandemic-related shifts in utilization of telehealth for clinical practice and student education have highlighted the advantages, disadvantages, and areas in need of additional study.

\section{Conclusions:}

Postpandemic telehealth care delivery will likely persist or expand. PMHNPs must advocate for high practice and education standards.

\section{Keywords}

telemental health, psychiatric nursing practice, nursing education graduate

\section{Introduction}

On March 11, 2020, the World Health Organization declared COVID-19 a global pandemic (Jack et al., 2021; World Health Organization, 2020). To reduce disease transmission, physical distancing measures and stay-at-home orders were enacted. As a result of these measures, psychiatric clinical care rapidly shifted to telehealth platforms (Chen et al., 2020). Telehealth is broadly defined as using telecommunication technology in the provision of clinical health care (U.S. Department of Health and Human Services, 2020). While telehealth was used by mental health providers prior to the pandemic in limited capacities, usage of telehealth became a practice norm during the COVID-19 pandemic. In response, psychiatric mental health nurse practitioners (PMHNPs), who previously saw patients faceto-face, have adapted practice to telehealth platforms to meet patient needs. With the ongoing pandemic, there is a need to safely meet not only the mental health needs of patients but also plan for the future of clinical care and education as telehealth will likely persist postpandemic.

The availability of highly effective COVID-19 vaccines show promise that the end of the pandemic might be on the horizon. However, now that telehealth has become a routine part of PMHNP practice and student education, it is unclear if the return to a previous "normal" will occur. This change in clinical service provision and student clinical education warrants a deeper exploration and understanding. Therefore, we have provided a summary of the advantages, disadvantages, and new challenges of telehealth for PMHNPs during the COVID-19 pandemic. Additionally, we suggest recommendations for future research and policy for clinical practice and education beyond the pandemic.

\section{Advantages}

Prior to the COVID-19 pandemic, telehealth was largely utilized to meet the psychiatric needs of patients in rural and psychiatrically underserved communities. Infrequency of use prepandemic meant that telehealth was not often part of clinical training for PMHNP students. However, there has been an accelerated expansion of telehealth in clinical practice and education in response to COVID-19 physical 
distancing practices (Fiks et al., 2021). The increased use of telehealth during the pandemic has highlighted the advantages of telehealth for clinicians, patients, students, and educators.

Prior to the pandemic, insurance reimbursement for telehealth visits was limited resulting in restricted use of telehealth in PMHNP in practice. Previously, Centers for Medicare \& Medicaid Services (CMS) reimbursed for telehealth only under limited circumstances constraining the use of these platforms for many clinicians and patients. Improved insurance reimbursement during the pandemic has led to a rapid expansion of telehealth services (CMS, 2020). Under the 1135 new waiver of the Social Security Act, Medicaid and Medicare will pay for primary care office, hospital, and other visits delivered from a patient's home via telehealth (CMS, 2020). Improved insurance reimbursement related to the expansion of waiver 1135 reduced the prohibitive, prepandemic financial barriers to telehealth visits.

Patients receiving telehealth visits might experience improved access to care, improved interdisciplinary care coordination, and reduced risk of illness exposure (Coker et al., 2019). For patients with psychosocial complexities, such as financial strain related to child care costs or limited access to reliable transportation to the PMHNP office, telehealth visits provide an opportunity to access care without the stress and cost related to in-person visits (Coker et al., 2019). Telehealth visits decrease the risk of patient, clinician, and student exposure to respiratory illnesses that they would face with in-person care. This is particularly advantageous during peak communicable viral illness times such as the ongoing COVID-19 pandemic (Feemster et al., 2019). Telehealth platforms allow primary and specialty care providers to collaborate when face-to-face appointments have historically made this challenging (Schaffer et al., 2020).

PMHNP students are able to continue their clinical training with the use of telehealth when in-person clinical experiences are not an option. Students in psychiatrically underserved areas with limited local preceptor opportunities are now able to complete clinical hours with preceptors across the country using telehealth (Schroeder, 2020). These students are receiving expert mentorship and training that they can bring back to communities in need of mental health services. The ability of students to complete clinical hours via telehealth platforms during the pandemic has allowed access to preceptors previously geographically inaccessible for many students.

\section{Disadvantages}

Telehealth usage for mental health care is poised to increase given the highlighted benefits experienced during the pandemic-related expansions in use of these platforms. However, there are disadvantages to expansions of telehealth that should also be considered. Disadvantages include inequities in access to care, patient safety concerns, risk of privacy breaches, and ambiguity regarding efficacy for all clinical situations. Students and educators may not be adequately trained in the provision of clinical education via telehealth platforms. Balancing the advantages of telehealth with lessons learned during the COVID-19 pandemic regarding the disadvantages of this care modality are key to driving future policy, practice, and student education guidelines.

The nature of telehealth engagement requires the use of telecommunication devices and access to the internet. Lack of reliable internet is a threat to health equity with telehealth care delivery. Disparities in access to broadband internet required for telehealth exist for racial minorities, older adults, rural residents, and those with lower levels of education and income (Pew Research Center, 2019). These 
disparities in access may drive mental health disparities among these groups in the future. Those patients who have access to reliable broadband internet may still be challenged with lack of familiarity with online platforms. The lack of familiarity with telehealth platforms and/or inequalities related to the financial burden of broadband internet access may result in feelings of defeat even before engagement in care with the PMHNP has begun.

Privacy and safety for the patient in the home setting of care warrants special consideration for the PMHNP when using telehealth platforms. We have seen dramatic increases in intimate partner violence during the COVID-19 pandemic (Evans, 2020). When care is offered in person, patient privacy and safety is traditionally protected "behind closed doors." The presence of physical privacy with inperson encounters can be protective for patients under threat of violence from a partner in the home. Introducing telehealth services in the home requires that patients have a safe space for discussion of health concerns to ensure that a similar "behind closed doors" privacy provision is maintained with telehealth visits. Inclusion of safety plans for patients at risk for interpersonal violence receiving telehealth care services are warranted (Jack et al., 2021).

Creating this "behind closed doors" privacy in a telehealth encounter is critical for patients to safely disclose stigmatizing experiences not only in individual but also in group therapy. Threats to confidentiality might be compounded with group therapy via telehealth (Brouwers, 2020). Each participant must ensure a private space for telehealth visits to ensure the privacy of all participants. The lack of physical space for telehealth visits, fear of experiencing stigma for engaging in mental health services by others in the home, or potential safety risks for those experiencing interpersonal violence may drive future disparities in access to care via telehealth.

Telehealth platforms may not be advantageous for all conditions and clinical scenarios. For example, patients in crisis or those experiencing active delusions or cognitive alterations might be at a disadvantage if care is only offered via telehealth encounters. Trust building and clinical assessments might be hampered for these patients and situations. For other patients in therapeutic groups, engaging in telehealth groups versus in-person visits may also provide variable experiences (Waller et al., 2020; Wootton, 2016). These considerations may limit the range of services that can be provided via telehealth.

There are disadvantages to using telehealth in the training of PMHNP students. Training in the use of telehealth was not often incorporated into didactic coursework prior to the pandemic; thus, students may feel underprepared to provide care virtually proficiently (Springer et al., 2020; Traube et al., 2021). Telehealth as a learning platform may feel impersonal, especially for students who are utilizing this as an exposure to learning clinical skills and techniques (Henry et al., 2021). There is a potential loss of connectedness and inability to establish trust or forming a therapeutic relationship with a patient due to a loss of nonverbal cues (Roesler, 2017).

\section{New Challenges}

There are new challenges in PMHNP practice and clinical education related to the rapid adaptation and expansion of use of telehealth during the COVID-19 pandemic. While clinicians and patients enjoyed benefits of telehealth care platforms during the pandemic, we learned about new challenges related to health equity in care access and care of special populations such as those with disabilities related to 
increased utilization of these care platforms. Additionally, those PMHNPs providing training and mentorship to student nurse practitioners are now having to define and shape clinical education in virtual encounters. Telehealth has become integrated into the provision of mental health services, but PMHNPs face new challenges regarding patient safety, health equity, clinical practice, and clinical education during the pandemic and beyond as formal telehealth guidelines are lacking or formative.

While current licensing and insurance reimbursement guidelines adopted during the pandemic have allowed for PMHNPs to regularly utilize telehealth, many clinicians did not have previous training on best practices and standards of care specific to these care platforms. The American Psychiatric Association and the American Telemedicine Association developed best practices for telehealth health services in 2018 (Shore et al., 2018). However, the rapid transition to this care model may leave many PMHNPs uncertain of best practice and in need of additional training and mentorship in this care model (Finley et al., 2020). For example, PMHNPs have well established protocols and procedures for patient safety and managing the care of patients in crisis during in-person visits. When providing care via telehealth, PMHNPs must similarly develop clear procedures and guidelines to manage patients in crisis such as those experiencing suicidal ideation. As expansion of licensing rules may result in patients and PMHNPs being in different geographic areas, it is vital for safety protocols to consider the patient's location and local resources. This must include identifying local collaborators and resources that can be called on to ensure patient safety during crises (Luxton et al., 2014).

In addition to developing practice guidelines for patients in crisis, PMHNPs must consider the needs of special populations such as those with disabilities in telehealth protocols. Telehealth may present unique challenges in care equity for patients with disabilities. There is considerable heterogeneity in the disability community that may have differential impacts and experiences with telehealth mental health care (Valdez et al., 2021). For some individuals with mobility disabilities, the movement to telehealth may reduce the stress and burden of transportation and facilities lacking accommodations needed for ease of access. However, those individuals with sensory impairments, such as the visually impaired or the deaf community, may struggle to engage or connect with the PMHNP via telehealth platforms. Among individuals with cognitive impairments, telehealth visits might present an added stress and burden to care engagement. As telehealth becomes integrated into care as a practice norm, there is a need for careful design and implementation considerations of these platforms to ensure equal access and care quality for individuals with disabilities (Valdez et al., 2021).

Beyond direct patient care protocols regarding safety and care of special populations, practice guidelines related to technology and privacy are needed for telehealth visits. Technology and platforms used for telehealth services rapidly evolved in response to increased use during the pandemic. The need to ensure that these platforms are Health Insurance Portability and Accountability Act (HIPAA) compliant is essential. However, how those privacy standards are established and maintained warrant clear description and outline in standard telehealth practice of care guidelines (Gabriel et al., 2018; HIPAA, 2021). Protecting patient privacy in the constantly evolving technologic frontier of telehealth is an ongoing challenge as this care model continues to be integrated into pandemic and postpandemic clinical practice for PMHNPs.

Many PMHNPs are taking on the added role of mentoring and training students using telehealth platforms. These mentors are providing vital training to students, but there is a paucity of guidance 
regarding how these experiences should occur for mentors, mentees, and patients. Educators need guidelines in best practice for mentorship using telehealth platforms for student clinical training (Johnson et al., 2020). Prior to coming to the clinical site, students should receive didactic course material in the provision of telehealth services (List et al., 2019). In the absence of didactic course materials in PMHNP curricula on telehealth platforms, clinical preceptors are training students during clinical rotation. This may result in variability of training experiences for students and an added burden to preceptors. Telehealth practice guidelines for educators and didactic coursework for students are needed to create uniformity in student training in telehealth services.

\section{Future Research and Policy}

PMHNP clinician, educator, and student experiences with telehealth during the COVID-19 pandemic must drive future PMNHP research and policy initiatives. Telehealth is a promising delivery model for mental health services; however, there is a paucity of research contributing to the literature from PMHNPs (Finley et al., 2020; Goldin et al., 2021). Previous literature regarding telehealth in practice and education examines telehealth use in physician practice rather than PMHNPs. PMHNPs differ from physician colleagues with regard to education, training, practice regulations, and licensure; therefore, research among PMHNPs is necessary (Finley et al., 2020; Schroeder, 2020).

Prior to the pandemic, physicians adopted a streamlined approach to compact licensure, and currently, 29 states participate in physician compact licensure agreements (Adashi et al., 2021). Efforts for similar compact licensure for advanced practice registered nurses is supported by leading nursing organizations, but legislation has not found similar traction in formalizing compact licensure as physician peers (Hahn, 2019). PMHNP licensing during the pandemic has allowed for loosening of state licensing restrictions. Postpandemic, PMHNPs must advocate for the care of their patients by demanding that governing bodies update licensing regulations inclusive of compact licensure to allow for continued access to vital mental health services.

Access to broadband internet has been named a social determinant of health during the pandemic because it is the necessary link to telehealth health services (Benda et al., 2020). However, health inequities in access to broadband internet exist (Smith \& Raskin, 2020). In identification of these care inequities, Healthy People 2030 has included increasing population-level broadband internet access as an objective (Office of Disease Prevention and Health Promotion, 2018). To advocate for the integration of telehealth in both education and practice, PMHNPs should insist that policymakers establish equitable access to technology infrastructure to support telehealth for both providers and patients (Kannarkat et al., 2020). Inadequate or lack of internet services limits the accessibility of services to those who may need the services the most. PMHNPs should advocate for state and federal legislation to address access to broadband internet, and that promotes health equity (Smith \& Raskin, 2020).

Inequities in telehealth care access also extend to persons living with disabilities. There is a risk that persons with disabilities might experience widening health disparities if careful consideration and adaptation of telehealth platforms to meet care needs is not considered. Future research exploring best practices for adaptation of telehealth care for persons with disabilities is necessary. PMHNPs must 
advocate for policies that support equitable access with appropriate adaptations of telehealth services for persons with disabilities.

PMHNPs must prepare for the predicted mental health fallout from the COVID-19 pandemic through clinical training and education of students (Estes \& Thompson, 2020). PMHNPs are charged with establishing formal telehealth education curriculum requirements to increase a telehealth-ready mental health workforce. PMHNP education on the use of telehealth platforms must be agile and responsive to the evolving needs of helping students meet the core competencies for entry into practice. Future practice guidelines governing student training in telehealth care provision could be driven by research among students who completed training during the pandemic. Surveys of PMHNP students after graduation to assess comfort and competency in the PMHNP role after receiving clinical training largely through telehealth patient encounters should be considered. Telehealth will likely be an enduring legacy of the COVID-19 pandemic; thus, PMHNP students must receive didactic and clinical training to effectively deliver care on this modality in the future.

\section{Conclusions}

The COVID-19 pandemic has been one of the most challenging times in modern history. Telehealth, however, has offered patients, clinicians, and students an opportunity to stay engaged during these unprecedented times. Improved reimbursement for telehealth and loosening of cross-state licensing for PMHNPs have reduced barriers to care during the pandemic. We have, however, seen threats to health equity grow during the rapid expansion of telehealth for those with limitations in access to broadband internet or for those with disabilities affecting care engagement. Students and educators have adapted rapidly to provide training and clinical experiences via telehealth. These rapid expansions in clinical care and education have brought to the forefront the need for research and policy to ensure high standards and equity in clinical care and education. PMHNPs have risen to the challenge of providing patient care and student education during the pandemic, and through lessons learned, they will be prepared to engage to expand and/or create policy and practice standards for telehealth services beyond the crisis.

\section{Author Note}

Jessica L Zemlak PhD, MSN, RN is currently an Assistant Professor at Marquette University College of Nursing in Milwaukee, WI.

\section{Author Roles}

JLZ and TR conceived the article and determined methods. All four authors contributed to researching, organizing, and writing the manuscript. JLZ wrote the abstract, introduction, new challenges, and conclusion sections. BV wrote the advantages section, TR wrote the disadvantages section, and PW wrote the future research and policy section. All authors reviewed the final draft prior to submitting for publication.

\section{Declaration of Conflicting Interests}

The authors declared no potential conflicts of interest with respect to the research, authorship, and/or publication of this article. 


\section{Funding}

The authors received no financial support for the research, authorship, and/or publication of this article.

\section{References}

Adashi, E. Y., Cohen, I. G., McCormick, W. L. (2021). The Interstate Medical Licensure Compact: Attending to the underserved. JAMA Journal of the American Medical Association, 325(16), 1607-1608. https://doi.org/10.1001/jama.2021.1085

Benda, N. C., Veinot, T. C., Sieck, C. J., Ancker, J. S. (2020). Broadband internet access is a social determinant of health! American Journal of Public Health, 110(8), 11231125. https://doi.org/10.2105/AJPH.2020.305784

Brouwers, E. P. M. (2020). Social stigma is an underestimated contributing factor to unemployment in people with mental illness or mental health issues: Position paper and future directions. BMC Psychology, 8(1), 36. https://doi.org/10.1186/s40359-020-00399-0

Centers for Medicare \& Medicaid Services . (2020). Medicare telemedicine health care provider fact sheet. https://www.cms.gov/newsroom/fact-sheets/medicare-telemedicine-health-careprovider-fact-sheet

Chen, J. A., Chung, W.-J., Young, S. K., Tuttle, M. C., Collins, M. B., Darghouth, S. L., Longley, R., Levy, R., Razafsha, M., Kerner, J. C., Wozniak, J., Huffman, J. C. (2020). COVID-19 and telepsychiatry: Early outpatient experiences and implications for the future. General Hospital Psychiatry, 66, 89-95. https://doi.org/10.1016/i.genhosppsych.2020.07.002

Coker, T. R., Porras-Javier, L., Zhang, L., Soares, N., Park, C., Patel, A., Tang, L., Chung, P. J., Zima, B. T. (2019). A telehealth-enhanced referral process in pediatric primary care: A cluster randomized trial. Pediatrics, 143(3), e20182738. https://doi.org/10.1542/peds.2018-2738

Estes, K. D., Thompson, R. R. (2020). Preparing for the aftermath of COVID-19: Shifting risk and downstream health consequences. Psychological Trauma: Theory, Research, Practice, and Policy, 21(S1), S31-S32. https://doi.org/10.1037/tra0000853

Evans, D. P. (2020). COVID-19 and violence: A research call to action. BMC Women's Health, 20(1), 249. https://doi.org/10.1186/s12905-020-01115-1

Feemster, K., Localio, R., Grundmeier, R., Metlay, J. P., Coffin, S. E. (2019). Incidence of healthcareassociated influenza-like illness after a primary care encounter among young children. Journal of the Pediatric Infectious Diseases Society, 8(3), 191196. https://doi.org/10.1093/jpids/piy023

Fiks, A. G., Jenssen, B. P., Ray, K. N. (2021). A defining moment for pediatric primary care telehealth. JAMA Pediatrics, 175(1), 9-10. https://doi.org/10.1001/jamapediatrics.2020.1881

Finley, B. A., Shea, K. D., Maixner, R., Slebodnik, M. (2020). Advanced practice registered nurses using synchronous telepsychiatry: An integrative systematic review. Journal of the American Psychiatric Nurses Association, 27(4), 271-282. https://doi.org/10.1177/1078390320939159

Gabriel, M. H., Noblin, A., Rutherford, A., Walden, A., Cortelyou-Ward, K. (2018). Data breach locations, types, and associated characteristics among US hospitals. American Journal of Managed Care, 24(2), 78-84.

Goldin, D., Maltseva, T., Scaccianoce, M., Brenes, F. (2021). Cultural and practical implications for psychiatric telehealth services: A response to COVID-19. Journal of Transcultural Nursing, 32(2), 186-190. https://doi.org/10.1177/1043659620973069 
Hahn, J. A. (2019). The perceptions and experiences of national regulatory nurse leaders in advancing the advanced practice registered nurse compact policy agenda. Journal of the American Association of Nurse Practitioners, 31(4), 255-

262. https://doi.org/10.1097/JXX.0000000000000135

Health Insurance Portability and Accountability Act . (2021). Healthcare data breach statistics. https://www.hipaajournal.com/healthcare-data-breach-statistics/

Henry, B. W., Billingsly, D., Block, D. E., Ehrmann, J. (2021). Development of the teaching interpersonal skills for telehealth checklist. Evaluation \& the Health Professions. Advance online publication https://doi.org/10.1177/0163278721992831

Jack, S. M., Munro-Kramer, M. L., Williams, J. R., Schminkey, D., Tomlinson, E., Jennings MayoWilson, L., Bradbury-Jones, C., Campbell, J. C. (2021). Recognising and responding to intimate partner violence using telehealth: Practical guidance for nurses and midwives. Journal of Clinical Nursing, 30(3-4), 588-602. https://doi.org/10.1111/jocn.15554

Johnson, D. S., Ling, A., Melino, K. (2020). Exploring readiness for teleprecepting in psychiatric mental health nurse practitioner training. Journal of the American Psychiatric Nurses Association, 27(2), 169-173. https://doi.org/10.1177/1078390320948127

Kannarkat, J. T., Smith, N. N., McLeod-Bryant, S. A. (2020). Mobilization of telepsychiatry in response to COVID-19: Moving toward 21st century access to care. Administration and Policy in Mental Health and Mental Health Services Research, 47(4), 489-

491. https://doi.org/10.1007/s10488-020-01044-z

List, B. A., Saxon, R., Lehman, D., Frank, C., Toole, K. P. (2019). Improving telehealth knowledge in nurse practitioner training for rural and underserved populations. Journal of Nursing Education, 58(1), 57-60. https://doi.org/10.3928/01484834-20190103-10

Luxton, D. D., Pruitt, L. D., Osenbach, J. E. (2014). Best practices for remote psychological assessment via telehealth technologies. Professional Psychology: Research and Practice, 45(1), 27-

35. https://doi.org/10.1037/a0034547

Office of Disease Prevention and Health Promotion . (2018). Healthy People 2030

framework. https://www.healthypeople.gov/2020/About-Healthy-People/DevelopmentHealthy-People-2030/Framework

Pew Research Center . (2019). Internet/broadband fact sheet. https://www.pewresearch.org/internet/fact-sheet/internet-broadband/

Roesler, C. (2017). Tele-analysis: The use of media technology in psychotherapy and its impact on the therapeutic relationship. Journal of Analytical Psychology, 62(3), 372-

394. https://doi.org/10.1111/1468-5922.12317

Schaffer, C. T., Nakrani, P., Pirraglia, P. A. (2020). Telemental health care: A review of efficacy and interventions. Telehealth and Medicine Today, 5(4). https://doi.org/10.30953/tmt.v5.218

Schroeder, R. A. (2020). Adaptation or revolution: Telemental health and advanced practice psychiatric nursing during COVID-19. Journal of the American Psychiatric Nurses Association.

Advance online publication. https://doi.org/10.1177/1078390320970638

Shore, J. H., Yellowlees, P., Caudill, R., Johnston, B., Turvey, C., Mishkind, M., Krupinski, E., Myers, K., Shore, P., Kaftarian, E., Hilty, D. (2018). Best practices in videoconferencing-based telemental health April 2018. Telemedicine and E-Health, 24(11), 827-

832. https://doi.org/10.1089/tmj.2018.0237

Smith, S., Raskin, S. (2020). Achieving health equity: Examining telehealth in response to a pandemic. Journal for Nurse Practitioners, 17(2), 214-217. https://doi.org/10.1016/j.nurpra.2020.10.001 
Springer, P., Bischoff, R. J., Kohel, K., Taylor, N. C., Farero, A. (2020). Collaborative care at a distance: Student therapists' experiences of learning and delivering relationally focused telemental health. Journal of Marital and Family Therapy, 46(2), 201-

217. https://doi.org/10.1111/jmft.12431

Traube, D. E., Cederbaum, J. A., Taylor, A., Naish, L., Rau, A. (2021). Telehealth training and provider experience of delivering behavioral health services. Journal of Behavioral Health Services \& Research, 48(1), 93-102. https://doi.org/10.1007/s11414-020-09718-0

U.S. Department of Health and Human Services . (2021). What is telehealth? https://www.hhs.gov/hipaa/for-professionals/faq/3015/what-istelehealth/index.html

Valdez, R. S., Rogers, C. C., Claypool, H., Trieshmann, L., Frye, O., Wellbeloved-Stone, C., Kushalnagar, P. (2021). Ensuring full participation of people with disabilities in an era of telehealth. Journal of the American Medical Informatics Association, 28(2), 389-

392. https://doi.org/10.1093/jamia/ocaa297

Waller, G., Pugh, M., Mulkens, S., Moore, E., Mountford, V. A., Carter, J., Wicksteed, A., Maharaj, A., Wade, T. D., Wisniewski, L., Farrell, N. R., Raykos, B., Jorgensen, S., Evans, J., Thomas, J. J., Osenk, I., Paddock, C., Bohrer, B., Anderson, K., Turner, H., Hildebrandt, T., Xanidis, N., Smit, V. (2020). Cognitive-behavioral therapy in the time of coronavirus: Clinician tips for working with eating disorders via telehealth when face-to-face meetings are not possible. International Journal of Eating Disorders, 53(7), 11321141. https://doi.org/10.1002/eat.23289

Wootton, B. M. (2016). Remote cognitive-behavior therapy for obsessive-compulsive symptoms: A meta-analysis. Clinical Psychology Review, 43, 103113. https://doi.org/10.1016/i.cpr.2015.10.001

World Health Organization . (2020). WHO director-general's opening remarks at the mission briefing on COVID-19. https://www.who.int/dg/speeches/detail/who-director-general-s-openingremarks-at-the-mission-briefing-on-covid-19 